\title{
DERECHOS EN CONFLICTO: UNA LEY ANTI-PIROPO EN ESPAÑA
}

\author{
Helena Rodemann Rounsevell \\ hrodemann1@gmail.com \\ Universidad de Deusto
}

Recibido: 25-01-2015

Aceptado: 26-04-2015

\section{Resumen}

Muchas personas creen que decirles comentarios a completas desconocidas es una forma de piropear y de elogiar inofensivamente su belleza. Por otra parte, a nivel político y social no se reconoce el acoso callejero como otra forma de violencia simbólica. Pero las reacciones cada vez más airadas al acoso motivan una conjetura acerca de la re-instauración de una ley antipiropo en el Estado español. Bajo la luz de la Constitución española, la pregunta plantea si una ley anti-piropo podría suponer un conflicto entre el derecho fundamental a la libertad de expresión de los hombres y los derechos a la integridad, privacidad, y seguridad de las mujeres. Palabras clave: Acoso callejero, piropos, violencia de género, derechos de las mujeres, derechos en conflicto, derechos constitucionales, libertad de expresión.

\begin{abstract}
Many people believe that complimenting unknown women is way of praising their beauty. In addition, on a political and social level, street harassment is not considered to be a form of symbolic violence. The increasing reactions to this type of harassment underline the necessity of a law prohibiting it. Under the light of the Spanish Constitution, the question remains as to whether such a law would imply a conflict between men's fundamental right to free speech and women's rights to integrity, privacy and security.
\end{abstract}

Keywords: Street harassment, "cat calling”, gender violence, rights in conflict, constitutional rights, free speech, women's rights. 
"The first time a man walked toward me, opened his mouth, began panting and jerked his crotch, I didn't feel the least bit affirmed or desirable. I did feel embarrassed, humiliated, furious-and helpless. It made me feel vulnerable and defenseless, as if I didn't really have any control over my own flesh.

(Bowman, 1993: 3)

\section{Introducción}

El hecho de que el trayecto diario de una mujer por la calle se convierta en una carrera de obstáculos o en la bajada de su mirada cuando se acerca a un grupo de hombres son, desafortunadamente, escenas muy frecuentes para muchas mujeres. En su documental Femme de la Rue, Sofie Peeters muestra, a través de cámaras ocultas, lo que vive cuando pasea por las calles de Bruselas. En el vídeo, graba como al caminar sola se expone a escuchar silbidos, gestos, burlas, e insultos como "puta”,"guarra” y “¿cuánto cobras?”. Desgraciadamente, su experiencia no es la única. Fairchild \& Rudman (2008) revelan que un $85 \%$ de mujeres dice haber sufrido este tipo de acoso. Pero a pesar de estos sucesos cotidianos iterativos, el acoso callejero es un tema bastante ignorado. Por una parte, muchas personas creen que decirles comentarios a completas desconocidas no constituye acoso, sino que es una forma de piropear y de elogiar inofensivamente su belleza. En su estudio realizado a 60 hombres de distintas ciudades, Benard y Schlaffer (1984) descubrieron que ninguno era consciente de que al escuchar "piropos" muchas mujeres se sienten acosadas. Por otra parte, a nivel político y social no se reconoce el acoso callejero como otra forma de violencia simbólica. Es visto como un mal menor, una expresión irremediable de la masculinidad. La cultura popular no cree que tenga ningún efecto real y que es una exageración tratarlo como si tuviera.

Pero hay autoras que discrepan. Cynthia Grant Bowman, catedrática de derecho de la Universidad de Cornell, (O'Neil, 2013) argumenta que los "piropos" ni son cumplidos ni son inofensivos. Son, rotundamente, un tipo de acoso porque son continuos y poco bienvenidos. Se entrometen en la vida de las mujeres e interrumpen su bienestar. Para situar en contexto, Bowman explica que el hecho ocurre en espacios públicos entre mujeres, las víctimas, y hombres, los autores. A continuación, los hombres evalúan la apariencia física de las mujeres 
ya sea para burlarse de ellas o para humillarlas a través de insultos, silbidos, guiños, proposiciones o comentarios, todos de carácter sexual (Chunn, 2011). Las mujeres no son inconscientes de la situación, y suelen reaccionar con enfado, miedo, o aparente nerviosísimo enmascarado como despreocupación (Benard y Schlaffer, 1984). Numerosos estudios demuestran los efectos nocivos de tales encuentros: dañan la autoestima y generan ansiedad, estrés, enfado, culpa, y depresión (Fairfield \& Rudman, 2008). Sólo con esto ya se demuestra que los mal llamados "piropos" son en verdad un acoso callejero que convierte los espacios públicos en ambientes hostiles para las mujeres.

Como reacción al documental de Peeters, Bélgica aprobó en 2014 una ley anti-piropo (Antiseksismewet) para intentar poner fin al acoso callejero. Curiosamente, a principios del siglo XX, el dictador Primo de Rivera también tuvo una iniciativa parecida. Prohibió el piropear a las mujeres porque lo consideraba demasiado vulgar. El Código Penal de 1928 luchaba por "el desarraigo de costumbres viciosas" como, por ejemplo "gestos, ademanes, frases groseras o chabacanas" (Manu, 2014). El incumplimiento de la norma suponía arresto de hasta 20 días o multas de hasta 500 pesetas. Pero la llegada de la República acabó con esta prohibición, y los hombres nuevamente pudieron salir a la calle para ofender a quien quisieran.

Las reacciones cada vez más airadas al acoso motivan una conjetura acerca de la reinstauración de una ley anti-piropo en el Estado español. Bajo la luz de la Constitución española, ¿qué problemas engendraría? Por un lado, hay quien opina que limitaría su libertad de expresión. Por otro lado, hay quien piensa que descargaría la tensión e inseguridad que sienten las mujeres cuando van por la calle. La pregunta plantea, pues, si una ley anti-piropo podría suponer un conflicto entre el derecho fundamental a la libertad de expresión de los hombres y los derechos a la integridad, privacidad, y seguridad de las mujeres.

\section{Libertad de Expresión}

Cualquier ley anti-sexista que regule el acoso callejero puede suponer varios problemas, sobre todo en relación a la libertad de expresión, un derecho universal y básico para cualquier sociedad democrática. El artículo 20.1 de la C.E. protege los derechos a "expresar y difundir libremente los pensamientos, ideas y opiniones mediante la palabra." Adicionalmente, “el 
ejercicio de estos derechos no puede restringirse mediante ningún tipo de censura previa" (España, 1978: 29317). Por lo tanto, cualquier restricción al pensamiento o a la expresión es autoritaria, déspota y anti-democrática. El jurista mejicano Miguel Carbonell (2011) añade que "una característica que parece estar presente en cualquier sistema autoritario es el control sobre lo que pueden y no pueden decir los ciudadanos. Por eso es que el grado de libertad de expresión que se disfruta en un país suele ser un indicador bastante fiable del avance democrático que se ha alcanzado" (par.1). En una democracia, prevalece la soberanía individual a pensar y a decir lo que se quiere, independientemente del contenido del mensaje. Eso sí, existen ciertas limitaciones, sobre todo si la expresión provoca daño serio o peligro inminente como, por ejemplo, las amenazas de muerte. En ese caso, la libertad de expresión debería ser regulada.

En cuanto a las mujeres, ya existen medidas de protección contra el acoso hacia ellas. En el estado español, la Ley de Igualdad del 2007 recoge que "constituye acoso por razón de sexo cualquier comportamiento realizado en función del sexo de una persona, con el propósito o el efecto de atentar contra su dignidad y de crear un entorno intimidatorio, degradante u ofensivo" (España, 2007: 12614). Pero muchos hombres tienen la certeza de que llamarla "tía buena" o "culo bonito" a una mujer no le causa ningún mal. Llamarle "guapa" no supone un peligro inminente, y "morena," u "ojazos" no se consideran insultos vejatorios. Por lo tanto, no se debería regular el poder comentar la apariencia de las mujeres.

Por otra parte, crear una ley que limita la libertad de los hombres de decirles cosas o silbarles a las mujeres por la calle supondría una confusión. Si se trata de un insulto explícito, queda claramente definido como injuria. Pero en el caso de no serlo, ¿qué exáctamente se prohibiría? ¿Silbar? ¿Llamarle 'guapa' a alguien? El coordinador del Centro de Ley Discriminatoria de la Universidad de Leuven, Jogchum Vrielink, advierte que este tipo de ley afectaría interacciones diarias básicas. El ligar, por ejemplo, podría interpretarse como sexista si una persona se refiriera a la otra como "atractiva," pues esto la reduciría a su dimensión sexual. De igual manera, Vrielink (2004) comenta que muchas mujeres tendrán vetado hablar de los hombres de manera ofensiva, lo cual se les haría muy difícil rebelar contra el status quo sexista, porque muchas veces rebelarse requiere lenguaje no sutil. La creación de esta ley en España crearía esta confusión ya que la definición podría ser arbitraria y subjetiva, por lo tanto, injusta. 
En conclusión, una ley que limitara la expresión "inocua” de piropear sería, pues, anticonstitucional y anti-democrático. La libertad de expresión es fundamental, y "no es una libertad más que pueda ponerse en la balanza al lado de otras libertades posibles para pesarla y contrapesarla con ellas, prevaleciendo en unos casos y quedando limitada en otros... No es una entre otras libertades, sino el fundamento de todo el orden político" (Laporta, 1997:14).

\section{Derecho a la Integridad}

Sin embargo, muchas mujeres reprochan los "piropos" por inhibir tres de sus derechos fundamentales: la integridad, la privacidad, y la seguridad. Cuando los hombres les dicen comentarios con tono sexual se suelen sentir acosadas y, como dijo el filósofo Hegel, es una violación de la libertad no poder ir a donde uno quiera por culpa de unas restricciones externas (Benard y Schlaffer, 1984). El acoso priva a las mujeres de estos tres derechos delimitados en la Constitución. Por lo tanto, los “piropos” podrían ser recriminados y denunciables.

La escena típica donde ocurre este acoso suele ser el mismo: una mujer entra o pasa por un lugar público y es asaltada por algún hombre que comenta sobre su aspecto físico y sobre todo, sobre su potencial sexual. Bajo esta perspectiva, el acoso callejero viola la integridad porque cosifica y deshumaniza a las mujeres al convertirlas en objetos de placer para los demás. Cuando un hombre opina sobre el cuerpo de una mujer, la reduce a un objeto sexual público apto para el juicio y escrutinio de los demás espectadores. Rara vez lo hace con la intención de hacerla sentir bien. Más bien al contrario, lo hace para humillar, avergonzar, y burlarse de ella. Al llamarla "guapa" o "tía buena" declara que tiene el derecho a tratarla como otro bien encontrado en la vía pública porque la juzga como juzga la utilidad de una papelera, la comodidad de un banco, o la limpieza de un parque. Su cuerpo es un objeto más, y él es el sujeto que lo controla. Pero las mujeres no son objetos, son personas, y tienen el derecho a ser tratadas como tal. Su humanidad consiste no sólo en sus cualidades físicas sino también en sus capacidades intelectuales, emocionales, sociales, laborales, etc. Por lo tanto, tienen el derecho a ser respetadas en su totalidad de forma íntegra. La C.E. delinea que toda persona tiene derecho a esta integridad "física y moral, sin que, en ningún caso, puedan ser sometidos a tortura ni a penas o tratos inhumanos o degradantes" (España, 1978: 29316). También "se garantiza el 
derecho al honor" [...] y a la propia imagen" (29317). La deshumanización de los "piropos" daña esta integridad moral de las mujeres, y la consecuencia es una cosificación que asocia el cuerpo con la humillación y vergüenza (Bowman, 1993). Por lo tanto, el "piropo" callejero debería estar ilegalizado porque objetiviza, deshumaniza, y, consecuentemente, afecta el derecho a la integridad moral de la persona receptora.

\section{Derecho a la Privacidad}

Otro derecho constitucional de las mujeres es el derecho a la intimidad personal (España, 1978: 29317). Pero aunque lo defienda la Constitución, la privacidad de las mujeres queda infringida en el momento que los hombres deciden acosarlas. Por ejemplo, cuando una mujer pasea por la calle pensando en sus preocupaciones, tareas, u obligaciones, ve interrumpida esa conversación interna y privada por el comentario inesperado de algún hombre a su alrededor. La antropóloga Micaela di Leonardo (Bowman, 1993) añade que no sólo se desvía su atención, también se interrumpe el trayecto y la libertad de pasear porque, acompañando el comentario, el hombre invade el espacio personal de ella al acercarse físicamente. Por lo tanto, su derecho a estar sola desaparece y el hombre que la acosa le roba la oportunidad de pasear, correr o pedalear en paz. Su cuerpo deja de ser sólo suyo porque queda expuesto a un escrutinio provocado por la creencia de su pertenencia a los hombres.

Ellos la hablan, tocan, o rozan sin ningún consentimiento previo y sin esperar respuesta a cambio. Hay hombres que incluso se exponen o se masturban delante suyo, independientemente de cómo sus actos influyen en la mujer. Como resultado, la mujer se ve obligada a interactuar con el acosador, ya sea respondiendo o fingiendo que le ignora. De cualquier manera, se impone una alteración de sus pensamientos y andares, y el espacio debidamente suyo deja de ser personal para que en su lugar sea invadido por un desconocido. El acoso restringe la movilidad física y geográfica porque muchas mujeres deciden alterar sus rutas o simplemente no salir a la calle para evitar ser acosadas. Es una vulneración de privacidad el no poder ir ni acceder a los lugares donde una quiera en paz, y el hombre que 
interrumpe con "piropos" los espacios mentales y físicos de esa mujer viola su derecho fundamental a la privacidad.

\section{Derecho a la Seguridad}

En la C.E., toda persona también tiene derecho a la seguridad (España, 1978: 29317), que la Real Academia Española define como la cualidad de ser "libre y exento de todo peligro, daño, o riesgo." Es decir, cada mujer debería gozar de una vida libre de violencia y miedo. Sin embargo, los "piropos" no consentidos infringen ese derecho porque inculcan lo contrario: inseguridad y temor. En la revista Pikara, la periodista June Fernández (2013) recalca que "las mujeres, por el hecho de serlo, estamos expuestas al (...) riesgo de ser agredidas física y sexualmente. Esto nos hace sentir vulnerables, expuestas, nos recuerda que la calle aún no es nuestra." Fairfield y Rudman (2008) descubrieron que las mujeres sienten mayor miedo por culpa de las "micro-victimizaciones" y "micro-agresiones" cotidianas de las miradas, silbidos, y comportamientos de los hombres. Estas experiencias las socializa a ser más temerosas y más perceptivas del peligro, sobre todo de la violencia sexual, porque las recuerda que en cualquier momento podrían ser agredidas.

De hecho, Bowman (1993) enfatiza que el acoso suele preceder la violencia ejercida. Muchos hombres lo utilizan para seleccionar a sus siguientes víctimas. Otras veces, las mujeres se exponen a más violencia si responden de manera desafiante al acoso. Holly Kearl y Lauren Taylor (2014), las primeras mujeres en redactar un informe nacional en Estados Unidos sobre el acoso callejero, cuentan las consecuencias a las que se enfrentan muchas mujeres que no toleran los “piropos." Tugce Albayrak, por ejemplo, murió después de que un hombre la pegó mientras defendía a dos adolescentes que estaban siendo acosadas. En octubre del 2014, un hombre apuñaló y mató a una adolescente egipcia que intentaba parar un acoso. En Detroit, un hombre disparó y mató a una mujer que se negó en darle su número de teléfono. En resumen, el acoso no sólo es violencia simbólica y psicológica sino que a veces llega a ser física también. La amenaza de la violencia esta muy interiorizada en la conciencia de las mujeres, y el acoso callejero las recuerda que debería seguir estándolo. Genera un miedo paralizante que inhibe rotundamente su sensación de seguridad. Pero también las pone en peligro porque refleja una 
creencia de la dominación masculina sobre la femenina que puede llegar a manifestarse físicamente. En conclusión, el acoso callejero infringe el derecho a la seguridad porque las daña y las expone al riesgo de violencia psicológica y física.

\section{Conclusión}

Indudablemente, el acoso perpetuado en las calles refleja un desequilibrio que coloca a las mujeres bajo una dominación masculina que las caracteriza como objetos sexuales. Esto apunta a un problema más grande: una desigualdad sistemática que discrimina a las mujeres por el simple hecho de serlo. Desafortunadamente, muchas mujeres creen que este tipo de violencia es incontrolable. Como consecuencia de esa desigualdad, algunas hasta profesan que les gusta. Otras opinan que si se contradice sólo generaría más violencia. Como resultado, muchas adoptan una resignación derrotista y creen que la solución es ignorarlo o pelearlo en vano. Leonardo (1981) subrayó que esta actitud quizás se deba a la falta de voluntad para reconocer su propia impotencia ante la situación. Otras mujeres, sin embargo, se aferran a su fuerza y toman iniciativas como Stop Street Harasssment, Hollaback y Everyday Sexism como herramientas para combatir el sin fin de obscenidades a las que se enfrentan las mujeres cuando salen a la calle. Ofrecen un espacio de solidaridad, sororidad, y empoderamiento; todos elementos fundamentales para obtener una igualdad que deje de encasillar a las mujeres como invasoras de un espacio público indebidamente suyo.

Después de la II Guerra Mundial, Eleonor Roosevelt visibilizó la perspectiva androcéntrica tradicionalmente aplicada a las leyes y normativas y subrayó la importancia de los derechos humanos en vez de, solamente, los derechos de los hombres. Bowman (1993:2) también enfatiza que las leyes trivializan o simplemente ignoran los eventos que tienen un efecto profundo sobre la conciencia de las mujeres. Aunque las leyes en el estado español reconozcan la existencia del acoso sexual y del acoso por razón de sexo, no mencionan el acoso callejero. Si lo hicieran, junto a la iniciativa ciudadana podrían resultar ser una estrategia efectiva para combatirlo. A través de la obligatoriedad, motivarían a las personas a cuestionarse sus comportamientos de manera más rápida. Podrían también contrarrestar las 
actitudes machistas que subordinan a las mujeres en los espacios públicos. Sin embargo, también podría pasar que unas leyes de este tipo no tendrían una efectividad en la realidad de las personas. Es decir, la estipulación de una norma no significa que realmente se lleve a cabo. Por esta razón, lo ideal en España sería que tanto las iniciativas sociales como las leyes trabajasen conjuntamente para educar y transformar las actitudes machistas que toleran el acoso.

Una ley anti-acoso callejero podría restringir la libertad de expresión de los hombres, pero la no aplicación de la ley supondría la violación de los derechos fundamentales a la integridad, privacidad, y seguridad de las mujeres. El conflicto nace, pues, al colocar sobre una balanza la importancia de un derecho sobre otros tres, o vice versa. Bowman (1993) argumenta que utilitariamente es mejor priorizar tres sobre uno ya que produciría mayor beneficio. Pero quizás jerarquizar los derechos no sea necesario. En otras palabras, quizás el problema no debería estar en cómo medir la prioridad de estos derechos, sino en cómo generar leyes que protejan la dignidad de ambas partes sin tener que sacrificar los derechos de nadie.

\section{BIBLIOGRAFÍA}

- Benard, Cheryl, Schlaffer, Edit (1984): “The man in the street: why he harasses". En: Feminist Frameworks. New York: McGraw-Hill.

- Bowman, Cynthia Grant (1993): "Street harassment and the informal ghettoization of women". En: The Harvard Law Review, Vol. 106, pp. 517-580. Versión [en línea] Disponible en: $\quad$ http://scholarship.law.cornell.edu/cgi/viewcontent.cgi?article=1141\&context=facpub [03/01/2015]

- Carbonell, Miguel (2011): “Libertad de expresión y democracia”, [en línea] Disponible en: http://www.miguelcarbonell.com/escritos_divulgacion/Libertad_de_expresion_y democracia.s html [15/12/2014].

- Chhun, Bunkosal (2011): “Catcalls: protected speech or fighting words?”. En: Thomas Jefferson Law Review, Vol. 33, № 2, pp. 273-295. Versión [en línea] Disponible en: http://www.tjeffersonlrev.org/sites/tjeffersonlrev.org/files/33-02-03-Chhun-Final.pdf [03/01/2015]. 
- España (1978): “Constitución Española”. En: Boletín Oficial del Estado, 29 de diciembre de 1978, núm. 311, pp. 29313-29424. Versión [en línea] Disponible en: http://www.boe.es/boe/dias/1978/12/29/pdfs/A29313-29424.pdf [03/01/2015].

- España (2007): "Ley Orgánica 3/2007, de 22 de marzo, para la igualdad efectiva de mujeres y hombres". En: Boletín Oficial del Estado, 23 de marzo de 2007, núm. 71, pp. 12611-12645. Versión [en línea] Disponible en: http://www.boe.es/boe/dias/2007/03/23/pdfs/A1261112645.pdf [03/01/2015].

- Fairfield, Kimberly y Rudman, Laurie A. (2008): "Everyday stranger harassment and women's objectification". En: Social Justice Research, 21, 338-357. Versión [en línea] Disponible en: doi: 10.1007/s11211-008-0073-0 http://www.ocacchile.org/wpcontent/uploads/2015/01/Kimberly-Fairchild-Everyday-Stranger-Harassment-and-

Women $\%$ E2\%80\%99s-Objetification.pdf [10/12/2014].

- Fernández, June (2013): Lo que El País no quiso publicar sobre acoso machista en la calle. Versión en [en línea] Disponible en: http://www.pikaramagazine.com/2013/03/lo-que-el-paisno-quiso-publicar-sobre-acoso-machista-en-la-calle/\#sthash.yNYECDz5.dpuf. [15/12/2014].

- Kearl, Holly y Taylor, Lauren R. (2014): "How do you respond to street harassment? Here are some suggestions". En: Ms magazine, [en línea] Disponible en: http://msmagazine.com/blog/2014/12/09/how-do-you-respond-to-street-harassment-here-aresome-suggestions/ [27/12/2014].

- Laporta San Miguel, Francisco Javier. (1997): “El derecho a informar y sus enemigos”. En: Claves de Razón Práctica, no. 72, pp. 14-19.

- di Leonardo, Micaela. (1981): "Political economy of street harassment". En: Aegis: Magazine on Ending Violence Against Women, Summer, pp. 51-57. Versión [en línea] Disponible en: http://www.stopstreetharassment.org/wpcontent/uploads/2011/04/PoliticalEconomyofStHarassment.pdf [03/01/2015].

- Manu (2014): “Un Madrid sin piropos”, [en línea] disponible en: http://www.secretosdemadrid.es/un-madrid-sin-piropos/ [26/12/2014].

- O’Neill, Jarrah. (2013): “Gender in public space: policy frameworks and the failure to prevent street harassment". En: Woodrow Wilson School of Public and International Affairs. Princeton University. Versión [en línea] Disponeble en: http://www.stopstreetharassment.org/wp-content/uploads/2011/04/JarrahONeill_thesis.pdf [26/12/2014].

- Vrielink, Jogchum. (2014): “No sexist speech please, we're Belgian”, [en línea] Disponible en: http://www.spiked-online.com/freespeechnow/fsn article/no-sexist-speech-please-werebelgian\# [15/12/2014]. 